\title{
Martina Damiani
}

\section{La centralità politico-culturale di Venezia negli scambi epistolari del primo Cinquecento}

Izvorni znanstveni rad

Research paper

UDK 655:821.131.1-6Aretino, P.>(450.341)

https://doi.org/10.32728/tab.17.2020.4

Nel Cinquecento, Venezia ha un ruolo centrale nella tipografia e un primato importante per quanto riguarda gli epistolari in volgare, con la pubblicazione, nel 1538, del primo libro di Lettere di Pietro Aretino che ha avuto ben dodici edizioni in soli due anni. Dalle Lettere traspare la fitta rete di contatti che l'autore era riuscito a stabilire (da Venezia) con diversi personaggi di spicco della scena politica e culturale (italiana e internazionale). Si prenderanno in esame gli accordi presi con alcuni editori e tipografi attivi a Venezia, tra cui Francesco Marcolini, per mettere in evidenza la sua strategia di autopromozione. L'ultima parte si soffermerà sulle critiche aretiniane nei confronti degli imitatori delle Lettere e sul trattamento di favore riservato invece a Paolo Manuzio, quale prolifico autore, curatore ed editore di epistolari.

Parole chiave: libri di lettere, tipografia veneziana, Pietro Aretino, Francesco Marcolini, Paolo Manuzio

\section{VENEZIA NELLE LETTERE DI PIETRO ARETINO}

Per spiegare il ruolo centrale che ricopre Venezia nelle epistole di Pietro Aretino, bisogna far innanzitutto riferimento al modo in cui questo letterato è giunto nella città lagunare. La scelta di spostarsi da Roma, dove era al servizio di Clemente VII, arriva nel 1525 quando Aretino rischia la vita dopo essere stato accoltellato da un sicario. Non sentendosi più al sicuro e manifestando rancore per il comportamento incurante del Papa, passa nel 1527 definitivamente a Vene- 
zia. Grazie alla sua "fama di temibile polemista", riceve la protezione della Serenissima in cambio della promessa di promuoverne la magnificenza e la potenza mediante la scrittura. ${ }^{1}$ Riserva perciò, nelle sue Lettere, molti elogi a Venezia e già in un'epistola del 1528 (quindi ad appena un anno di permanenza nella città) esprime la volontà di non cambiare mai dimora: "l'animo mio è di starci sempre, che è pazzo chi non sa vivere in paradiso"2. Aretino non mancherà di lodare la nuova patria anche in altre sue opere, mostrandosi sempre particolarmente attento a non offendere i suoi ospiti veneziani ${ }^{3}$; nella commedia La Cortigiana del 1534 , sottolinea che "i vertuosi sono ivi, e la gentilezza de le persone è a Vinegia", mentre rappresenta Roma dominata dalla "villania" e dall'“invidia" (Cort., III, 7, 135). Allo stesso modo di Aretino, un personaggio della pièce decide di abbandonare la corrotta corte romana in favore della città lagunare: "lo me ne andrò forse a Vinegia, perché solamente in Vinegia la giustizia tien pari le bilance; [...] e certamente ella è la Città Santa e il Paradiso terrestre" (Cort., III, 7, 134). Agli occhi di questo personaggio, che rappresenta l'alter ego dello scrittore, Roma non merita di essere considerata la Città Santa (tantomeno dopo le violenze subite durante il Sacco del 1527), mentre lo diventa Venezia: unico Paradiso terrestre dove regna la giustizia e la pace ${ }^{4}$.

Aretino loda il sistema veneziano e cerca di ingraziarsi soprattutto i potenti ottenendo, sin dai primi anni a Venezia, addirittura l'appoggio e la protezione del doge Andrea Gritti (doge in carica dal 1523

1 Paul Larivaille, Pietro Aretino, Salerno Editrice, Roma 1997, pp. 75, 153.

2 Pietro Aretino, Lettere, Libro Primo, a cura di Paolo Procaccioli, Salerno Editrice, Roma 1997, lettera 11, Venezia 8 giugno 1528, p. 67.

3 Nonostante pubblichi le sue opere a Venezia, le ambienta sempre altrove perché contengono alla loro base un'aspra critica sociale e morale che non poteva riguardare la città che gli aveva dato rifugio. Per quanto riguarda, ad esempio, le sue commedie, Aretino ambienta La Cortigiana e La Talanta a Roma, II Marescalco a Mantova e Lo Ipocrito a Milano. Cfr. Paul Larivaille, Pietro Aretino, cit., p. 200.

4 Mentre Venezia è identificata con il Paradiso, Roma sembra l'Inferno, come si legge in una lettera del 1537: "Io, fratello non veggo andar mai alcun de i miei amici a starsi a Roma, ch'io non pianga la lor disgrazia, più che s'andassero a la sepoltura, perché ne la fossa si sepelliscano i morti e ne la corte i vivi. E quel dolore, che si averia sapendosi che un fratello fusse ne lo Inferno, s'ha di coloro che vivono ne la crudeltà di così fatto abisso". A Venezia tale tranquillità è possibile, per l'autore, grazie alle leggi che garantivano l'ordine: “I'Eccellenze di cotanta Repubblica [...] comandando cose oneste e vetando le disoneste per via del costume [...] ha creato leggi castissime il cui ordine frena l'audacia de i rei, e assicura l'innocenza de i buoni”. Pietro Aretino, Lettere, Libro Primo, cit., lett. 284, Venezia 10 dicembre 1537, p. 393. 
al 1538$)^{5}$. Tale protezione ha sicuramente favorito la reputazione e l'importanza dello scrittore che ricambia tale benevolenza elogiandolo nelle proprie opere. Ricorderemo, a tale proposito, la canzone In Laude di Venetia in cui si ritiene Andrea Gritti capace il governare in maniera esemplare non soltanto la città lagunare ma anche "due Venetie, tre Rome e quattro Egitti”.

In una lettera scritta al Doge nel 1530, Aretino elenca quali sono i pregi di vivere a Venezia: "qui non ha luogo il tradimento, qui il favore non può dar torto al diritto, qui non regna la crudeltà de le meretrici, [...] qui non si ruba, qui non si sforza e qui non si ammazza". Infine passa a esaltare la bellezza della città che "risplende ne le nobiltà, ne le magnificenze, nel dominio, negli edifici, ne i tempi, ne le case pie"7. Tra le case della laguna Aretino riserva uno spazio particolare a palazzo Bolani, sua dimora per più di vent'anni. In una lettera (datata ottobre 1537) si sofferma sulla splendida residenza collocata, come egli stesso evidenzia, nel "più degno lato ch'abbia il canal grande", da dove poteva godere "de la più bella strada e de la più gioconda veduta del mondo"'. Questo riferimento conferma la posizione privilegiata che l'autore aveva nell'ambiente veneziano, tanto da permettersi un'abitazione del genere dove ospitava personaggi importanti (tra cui il grande Tiziano ${ }^{9}$ ), molti dei quali sarebbero divenuti soggetti e destinatari delle sue lettere.

Da Venezia, Aretino intrattiene corrispondenze con i più importanti signori del tempo come traspare dai nomi altisonanti presenti

5 La protezione del Doge non passa inosservata neppure a Roma, come risulta da una lettera scritta nel 1531 dal segretario di Clemente VII, Giovan Battista Sanga: "il Serenissimo Gritti, come Principe di somma bontà e religione, si è mosso in atto di carità e di amore, virtù proprie degne del suo animo e de la sua prudenzia, a chiamarvi a sé". Vedi Lettere scritte a Pietro Aretino, Libro I, Tomo I, a cura di Paolo Procaccioli, Salerno Editrice, Roma 2003, p. 42.

6 Pietro Aretino, In Laude di Venetia, in Scritti di Pietro Aretino nel Codice Marciano, It. XI 66 (=6730), Danilo Romei (a cura di), in http://www.nuovorinascimento.org/n-rinasc/testi/pdf/aretino/scritti.pdf, vedi ottava 21, vv. 7-8, p. 44.

7 Pietro Aretino, Lettere, Libro Primo, cit., lett. 2, Venezia 1530, p. 50.

8 Nella stessa lettera definisce "Vinezia la Papessa d’ogni altra cittade” e loda la nobiltà dei suoi vicini di casa e, in generale, la ricchezza dei veneziani. Pietro Aretino, Lettere, Libro Primo, cit., lett. 212, Venezia 27 ottobre 1537, p. 301.

9 Per l'amicizia con Tiziano, al quale Aretino scrive quarantaquattro lettere, vedi Paul Larivaille, Pietro Aretino, cit., pp. 290-292. 
tra i suoi mittenti ${ }^{10}$. In molte lettere, si presenta come una specie di ambasciatore interessato a instaurare una politica positiva a vantaggio della Serenissima, mettendo la propria penna al servizio della città ${ }^{11}$.

In particolare nel Libro Primo, Aretino espone a vari destinatari il problema dell'avanzata turca nel Mediterraneo, favorendo così la politica veneziana ${ }^{12}$. Il suo intervento si colloca non a caso nel 1536, anno in cui viene firmato un trattato tra la Francia e la Turchia, che rischiava di compromettere seriamente il predominio commerciale di Venezia ${ }^{13}$. In questa circostanza, Aretino scrive all'imperatore Carlo V, presentandolo come l'unico capace di fermare l'avanzata Turca e porre "le catene de la servitù a le braccia di tutto l'Oriente"14. Successivamente, si rivolge pure al sovrano francese, Francesco I, nel tentativo di dissuaderlo da tale alleanza, consigliandogli di seguire l'esempio dei veneziani impegnati nella lotta contro il turco:

La Maestà Vostra ha inteso la religiosa, l'ottima e la magnanima deliberazione fatta dal debito e dal costume dei religiosi, ottimi e magnanimi Veniziani. Voi sapete come essi, sprezzando le lor

10 A proposito dei suoi mittenti, nella lettera dedicatoria al cardinale Innocenzo del Monte (nipote di Giulio III), il suo editore, Francesco Marcolini, affermerà: "vi parrà miracolo, vedendo la moltitudine de le Cortesie usategli quasi da tutti i Magnati; che così testimoniarebbesi da le pistole, se appresso di sé avesse tenuto cura de la moltitudine da lui a dì per dì ricevute da Cavalieri, da Signori, da Conti, da Marchesi, da Duci, da Principi, e da ciascuna sorte de personaggi grandi e Madonne. Di Francesco e di Enrigo, I'uno d'Inghilterra Re, e l'altro di Francia Sire, e di alcune Madame e Reine". Vedi Lettere scritte a Pietro Aretino, cit., Venezia ottobre 1551, p. 33. Considerando il ricco scambio epistolare con personalità di spicco del mondo politico di allora e il tentativo di intrattenere rapporti diplomatici con diverse nazioni è stata avanzata addirittura l'ipotesi che Aretino sia stato un agente informatore delle autorità veneziane, ciò spiegherebbe la protezione ricevuta da parte del Doge. Cfr. Paul Larivaille, La vita quotidiana delle cortigiane nell'Italia del Rinascimento. Roma e Venezia nei secoli XV e XVI, Rizzoli, Milano 1987, p. 186.

11 Cfr. Paul Larivaille, Pietro Aretino, cit., p. 153.

12 Aretino affronta il problema dell'avanzata turca anche in altre opere e in particolare in ben tre delle sue commedie (La Cortigiana, La Talanta e Lo Ipocrito). Cfr. Martina Damiani, Il confronto di approcci diversi alla minaccia straniera nelle commedie rinascimentali, in Comico e tragico nella vita del Rinascimento, Atti del XXVI Convegno Internazionale (Chianciano Terme-Pienza, 17-19 luglio 2014), a cura di Luisa Secchi Tarugi, Franco Cesati Editore, Firenze 2016, pp. 323-328, 330-331.

13 Cfr. Paul Larivaille, Pietro Aretino, cit., pp. 153, 216.

14 Aretino ricorda in questa lettera la liberazione di ventimila cristiani dalla schiavitù del turco, avvenuta durante una spedizione nel maggio del 1535. Vedi Pietro Aretino, Lettere, Libro primo, cit., lett. 66, Venezia 4 giugno 1536, p. 125. 
ricchezze in Levante, i tesori che ne traevano, la perdita del sangue istesso e le inaudite offerte del Turco, hanno, insieme con Pietro e con Cesare [con il papa e l'imperatore], rivoltate le forze del mare e de la terra in servigio di Cristo ${ }^{15}$.

Aretino non perde l'occasione di celebrare Venezia per la sua cristianità, considerando nobile il modo in cui avrebbe messo da parte il proprio interesse per andare in soccorso alla fede ${ }^{16}$. In realtà Venezia entrò nella lega Santa perché i turchi rappresentavano un impedimento alla sua attività mercantile, motivo che il Nostro sicuramente conosce ma di cui, ovviamente, non fa cenno nelle lettere (per presentarlo come un gesto disinteressato ${ }^{17}$. Quando, nel febbraio del 1538, viene stipulato il patto di alleanza antiturca, firmato dal Papa, dall'imperatore e da Venezia, si innalza indirettamente pure il "prestigio diplomatico dell'Aretino" che aveva sostenuto e incoraggiato tale unione ${ }^{18}$.

Grazie alle sue strategiche intercessioni presso i potenti e ai legami stabiliti con personaggi di spicco del panorama politico e culturale, questo singolare scrittore riesce a catturare l'attenzione e la curiosità del pubblico, sfruttando al meglio la fiorente attività tipografica veneziana.

15 Pietro Aretino, Lettere, Libro Primo, lett. 195, Venezia 18 settembre 1537, p. 280 (corsivi nostri). Nel primo libro, sono quattro le lettere indirizzate a Francesco I (mentre ben sei quelle scritte a Carlo V).

16 Lo stesso concetto è ribadito anche nella lettera successiva: "per interesse de la sua fede si move l'incredibile religione de la bontà Veneziana". Pietro Aretino, Lettere, Libro Primo, cit., lett. 198, Venezia 19 settembre 1537, p. 285.

17 In un'altra lettera sottolinea esplicitamente il disinteresse economico della città: "Che bel vanto darà la fama per tutto il mondo e in ciascun secolo a Venezia, avendo ella per Giesù disprezzato il sangue e le ricchezze!” Pietro Aretino, Lettere, Libro Primo, cit., lett. 200, Venezia 22 settembre 1537, p. 287.

18 Giovanni Aquilecchia, Nuove schede di italianistica, Salerno Editrice, Roma 1994, p. 82. II 5 giugno 1538, lo scrittore intesta una lettera proprio a Paolo III per ricordare la partecipazione del Papa al convegno di Nizza col proposito di ristabilire la pace tra i sovrani Carlo V e Francesco I, alleanza basilare per far indietreggiare il turco dalla conquista degli stati cristiani. In modo indiretto, Aretino sembra qui far riferimento alla propria intercessione in favore di tale alleanza, iniziata ben due anni prima. Si riporta un breve passo della lettera indirizzata a Paolo III: "Padre beatissimo, la cagione, che vi ha mosso da Roma a Nizza, è la più gloriosa di quante ne accorsero mai da che i pontefici fur vicari di Cristo. Ella è il decoro del grave dei vostri anni, l'ornamento del sacro del vostro grado e il diadema del santo de la vostra vita". Pietro Aretino, II secondo libro delle lettere, Parte prima, a cura di Fausto Nicolini, Laterza, Bari 1916, lettera 344, Venezia 5 giugno 1538, p. 23. 


\section{LA TIPOGRAFIA DI FRANCESCO MARCOLINI}

Nel 1538, l'editore e tipografo Francesco Marcolini da Forlì (attivo a Venezia dal 1535) pubblica il primo libro di Lettere di Pietro Aretino ${ }^{19}$. I due sono legati da un rapporto di fiducia e amicizia, come dimostrano alcuni scambi epistolari. Aretino nomina e ringrazia l'editore in diverse lettere, riportando pure i loro accordi. Apprendiamo così che vi era una diretta e attenta partecipazione dell'autore alle varie scelte tipografiche, come testimoniato dalla "vastità d'interventi in fase di stam$\mathrm{pa}^{20}$. Scopriamo inoltre che Aretino chiede al suo editore di stampare il primo libro di Lettere a proprie spese, promettendo di lasciargli tutto il guadagno ricavato dalla vendita dei libri, perché egli aspirava a molto di più e cioè alla generosità dei potenti: "lo voglio [...] che la cortesia dei principi mi paghi le fatiche de lo scrivere, e non la miseria di chi le compra". A Venezia muta la sua condizione di letterato in quanto non deve più sottostare ai voleri di un mecenate, ma vari potenti, per non essere attaccati dalla sua satira pungente, lo mantengono senza limitarlo nelle scelte letterarie. Diventa, in poco tempo, uno dei primi letterati a essere in grado di vivere in modo autonomo, facendo unicamente affidamento alla scrittura.

Per convincere il tipografo a stampare le sue epistole senza chiedergli un compenso, il Nostro manifesta l'intenzione di pubblicare in futuro ogni opera presso la tipografia di Francesco Marcolini: "di mano in mano sarete erede di ciò che mi uscirà de l'ingegno"21. All'editore poteva bastare questa promessa, visto che si trattava di uno scrittore molto prolifico (dal suo arrivo a Venezia si era fatto conoscere "sfornando" commedie e trattati con un ritmo frenetico ${ }^{22}$ ). Come si evince da questa concessione, in quel momento Aretino non poteva immaginare lo straordinario successo che avrebbe ottenuto il suo libro, con

19 Per l'attività di Francesco Marcolini in qualità di editore dei libri di Pietro Aretino, cfr. Paolo Marini, Gli "Scritti" di Francesco Marcolini a cura di Paolo Procaccioli, in Scaffale Aperto, VI, 2015, pp. 141-151.

20 Fabio Massimo Bertolo, Aretino e la stampa. Strategie di autopromozione a Venezia nel Cinquecento, Salerno Editrice, Roma 2003, p. 16.

21 Pietro Aretino, Lettere, Libro Primo, cit., lettera 1 (appendice), Venezia 22 giugno 1537, p. 513.

22 Aretino si vanta spesso della sua capacità di scrivere in breve tempo più opere e in una lettera ad Alessandro Piccolomini rivela: "ne le ore da me furate al sonno di forse venti notte, ho, come si sa, composto due commedie". Pietro Aretino, Lettera al Piccolomini in Tutto il teatro, a cura di Antonio Pinchera, Newton, Roma 1974, p. 371. 
ben dodici edizioni in soli due anni ${ }^{23}$, e così indirettamente contribuisce alla fortuna di Francesco Marcolini nel campo dell'editoria veneziana. Questi diventa, infatti, il primo editore ad aver pubblicato un libro di lettere in volgare, e non un libro qualsiasi, ma quello che può considerarsi un best seller dell'epoca.

In seguito, però, il Nostro attribuisce unicamente a sé stesso tutti i meriti visto che era stata sua l'idea di pubblicare dei libri di lettere, che rappresentavano effettivamente "una nuova tipologia libraria"24. Oltre a sottolineare il suo primato, Aretino biasima coloro che, subito dopo di lui, hanno iniziato a rendere pubblici i loro scambi epistolari25. II primo a imitarlo è Nicolò Franco che nel 1537 era entrato a suo servizio, diventandone segretario, proprio mentre il Nostro stava terminando di revisionare il primo libro di lettere in vista della pubblicazione. Nonostante l'iniziale stima reciproca, i due diventano presto rivali, soprattutto con la decisione del Franco di raccogliere le proprie epistole e di farle stampare a Venezia nel 1539 con il titolo di Pistole vulgari26. Pietro Aretino attaccherà il suo ex collaboratore per avergli copiato l'idea, anche se I'ha camuffata dietro a un titolo diverso:

Ecco: un Franco di Benevento, capitatomi inanzi ignudo e scalzo come andrà sempre, dopo i segnalati benefizi da me ricevuti, volse concorrer meco e per aver detto Pistole e non Lettre, ne va altero, quasi vincitor di quel ch'io sono. ${ }^{27}$

Dopo l'uscita di questo e di molti altri epistolari in volgare, Aretino, giunto nel 1546 al suo terzo libro, si chiede: "non meritano gli inven-

23 I cinque libri di Lettere successivi non conoscono lo stesso successo e solamente il secondo, pubblicato nel 1542, ha una seconda edizione. Ricordiamo inoltre che il terzo libro esce nel 1546, il quarto e il quinto nel 1550, mentre il sesto viene pubblicato postumo, nel 1557. Cfr. Amedeo Quondam, Aretino e il libro. Un repertorio, per una bibliografia, in Pietro Aretino nel Cinquecentenario della nascita. Atti del Convegno di Roma-Viterbo-Arezzo (28 settembre-1 ottobre 1992), Toronto (23-24 ottobre 1992), Los Angeles (27-29 ottobre 1992), Tomo I, a cura di Enrico Malato, Salerno Editrice, Roma 1995, p. 217.

24 Fabio Massimo Bertolo, Aretino e la stampa, cit., p. 13.

25 Pietro Aretino, Lettere, Libro Terzo, a cura di Paolo Procaccioli, Salerno Editrice, Roma 1999, lett. 26, Venezia novembre 1542, p. 38.

26 Nicolò Franco, Le pistole vulgari, Antonio Gardane, Venezia 1539.

27 Pietro Aretino, I/ secondo libro delle lettere, cit., lett. 493, Venezia 15 febbraio 1540, p. 215. Anche nel quarto libro introduce lo scrittore di Benevento in termini simili: "lo ignudo, e scalzo, e affamato Franco, imparò da me, già padron suo [...]". Pietro Aretino, Lettere, Libro Quarto, a cura di Paolo Procaccioli, Salerno Editrice, Roma 2000, lett. 271, Venezia dicembre 1547, p. 175. 
tori de le cose qualche poco di laude?"; spiega, infine, tale quesito autopromuovendosi: "le prime lettere che in lingua nostra siano state impresse, nascon da me"28. In questo preciso momento, il Nostro può avanzare tali affermazioni (e negare l'apporto del suo editore) visto che a stampare l'opera non è più Francesco Marcolini (che si era allontanato per un periodo da Venezia, trasferendosi a Cipro). Per la pubblicazione del terzo libro si affida a un altro tipografo, Gabriele Giolito de' Ferrari (che stamperà in seguito altre nove edizioni delle opere aretiniane, tre prime edizioni e sei ristampe ${ }^{29}$. Nel terzo libro troviamo ben nove lettere indirizzate a Marcolini, nelle quali non mancano cenni alla sfera lavorativa; Aretino si lamenta col suo primo editore della quantità di lettere che gli venivano recapitate ogni giorno da corrispondenti diversi (i quali si auguravano evidentemente di ricevere una risposta per vederla poi inserita nei volumi successivi): "Ho inteso il romore che fanno coloro che si dolgono del mio non rispondere mai al loro iscrivermi sempre". Pietro confida così al tipografo (e ai futuri lettori) di compiere una selezione delle lettere perché altrimenti: "le stampe non arebbon mai tanto spazio, che l'attendere ad altro che a imprimere l'opere mie"30. Nell'ultima epistola che, nel terzo libro, è indirizzata a Marcolini (datata settembre 1545), lo scrittore si rammarica che l'amico sia partito da Venezia senza neppure salutarlo. Gli confida, certo della sua comprensione, di non poter aspettare il suo ritorno per stampare il nuovo volume di Lettere (ormai concluso), ma di doversi affidare a un altro tipografo: "E perché io so, che sapete, che non posso vivere senza mangiare, non posso credere che non potendo prima che dopo più d'uno anno attendere a l'arte de l'imprimere volumi, abbiate a male la forza che mi costringe a commettere l'ultime cose mie al carattero d'altri" ${ }^{31}$. Non fa qui menzione a quale tipografo si sarebbe rivolto e non troviamo nel terzo libro neppure una lettera inviata al suo nuovo editore, Gabriele Giolito, per cui si può ritenere tale scelta realmente dettata dalla necessità del momento. Mentre Aretino non si fa scrupoli a cambiare editore e a smentire il contributo di Francesco Marcolini da Forlì nella "creazione" del primo libro di Lettere, egli mostrerà invece maggior rispetto nei confronti dei Manuzio.

28 Pietro Aretino, Lettere, Libro Terzo, cit., lett. 26, Venezia novembre 1542, p. 38. Lettera indirizzata all'amico e poeta Niccolò Martelli.

29 Per i riferimenti a Gabriele Giolito (che stamperà anche il sesto libro delle Lettere), cfr. Fabio Massimo Bertolo, Aretino e la stampa, cit., p. 116.

30 Pietro Aretino, Lettere, Libro Terzo, cit., lett. 98, Venezia agosto 1544, pp. 114-115.

31 Ivi, lett. 322, Venezia settembre 1545, p. 285. 


\section{PAOLO MANUZIO E LE RACCOLTE EPISTOLARI}

Pietro Aretino elogia la famiglia Manuzio in diverse occasioni; in un'epistola del novembre 1542, afferma che "i caratteri de le stampe d'Aldo sono simili a le perle" 32 , facendo probabilmente riferimento all'eleganza del carattere corsivo (detto anche aldino), adottato da quest'importante tipografo. Ricorda poi che l'innovazione di Aldo Manuzio è stata sfruttata da molti tipografi, estendendo la critica agli editori cinquecenteschi che si stavano "macchiando" di una seconda "colpa". Questi, infatti, visto il mercato favorevole, avevano copiato l'idea anche ad Aretino, dando avvio alla stampa di una quantità eccessiva di libri di lettere, "un tuono di pistole che spaventerebbe i fulmini che cascano dal cielo"33.

Da questa critica però sembra esonerato il figlio di Aldo Manuzio, Paolo, che aveva ripreso l'attività editoriale paterna nel 1533, pubblicando diversi libri di lettere. A causa della sua delicata posizione di forestiero, Aretino non poteva muovere alcuna critica alle persone più in vista di Venezia, tra cui la famiglia Manuzio. Non accuserà mai un tipografo veneziano di avergli rubato l'idea, come aveva invece fatto con Nicolò Franco (originario di Benevento), nonostante Paolo Manuzio abbia effettivamente basato gran parte della sua fortuna proprio sui libri di lettere. Nel 1542 esce la prima raccolta manuziana di epistole, dal titolo Lettere volgari di diversi nobilissimi huomini et eccellentissimi ingegni scritte in diverse materie (farà seguito il secondo libro nel 1545 e il terzo nel 1564) $)^{34}$. L'editore seleziona un numero elevato di lettere indirizzate ai personaggi più noti del periodo, tra cui Pietro Bembo, ma anche qualcuna spedita a Pietro Aretino che, visto il successo delle sue Lettere, non poteva certo mancare. La raccolta contiene soltanto due lettere scritte da Aretino, mentre sono ben dieci le epistole a lui indirizzate ${ }^{35}$. Ricordiamo che le Lettere scritte a Pietro Aretino saranno pubblicate appena

32 Pietro Aretino, Lettere, Libro Terzo, cit., lett. 26, Venezia novembre 1542, p. 38.

33 Ibidem.

34 Cfr. Tiziana Sterza, Paolo Manuzio editore a Venezia (1533-1561), in Acme, Annali della Facoltà di Lettere e Filosofia Università Statale di Milano, vol. LXI, II, 2008, p. 146.

35 Tra queste lettere, tre sono state scritte dal filologo Claudio Tolomei che in una missiva (datata Roma, 8 aprile 1541) loda “la fertilità dell’ingegno" di Pietro Aretino e la velocità con cui compone le sue opere: "che non prima ha fatto un bel frutto, ch'ella incomincia a sputar fuor novi fiore per produrre l'altro". Il primo libro esce dalla tipografia del Manuzio nel 1542, l'edizione che abbiamo consultato è invece posteriore. Paolo Manuzio (a cura di), Lettere volgari di diversi nobilissimi huomini et eccellentissimi ingegni scritte in diverse materie, Domenico Giglio, Venezia 1558, libro II, p. 19. Il terzo libro non contiene lettere scritte o indirizzate ad Aretino. 
nel 1551-52 in due libri, anche se alcune di queste erano già state stampate da Francesco Marcolini nelle riedizioni del primo libro: scelta fatta per differenziarle dalle altre edizioni in circolazione. Il successo del libro e la conseguente continua richiesta di altre copie, aveva portato cinque tipografi a puntare su questo letterato e a "scavalcare" Marcolini, stampando riedizioni non autorizzate dell'opera. Infatti, dalla prima edizione delle Lettere (1538) al 1542, anno di uscita del secondo libro, oltre alle tre ristampe di Marcolini, escono anche ben otto edizioni pirata ${ }^{36}$. Quest'attenzione degli altri tipografi veneziani nei confronti del primo libro di Lettere non poteva essere vista di cattivo occhio dall'autore visto che, come si apprende dalla corrispondenza col suo editore (precedentemente citata), aveva rinunciato all'introito delle vendite, mentre ogni riedizione contribuiva a diffondere l'opera e a incrementarne la fama. Per distinguersi da queste "copie", e vendere meglio il libro, Marcolini nelle sue riedizioni propone delle nuove lettere: venticinque nella prima ristampa del 1538 e altre quarantaquattro nella terza del 1542, molte di queste sono epistole indirizzate all'ormai celebre autore ${ }^{37}$.

Aretino farà riferimento alle lettere stampate dal Manuzio nel terzo libro, dimostrando la sua disapprovazione per alcune epistole tratte proprio da una riedizione delle Lettere. Queste sarebbero state pubblicate nella raccolta manuziana omettendo le parti, a suo avviso, più importanti, quelle cioè che maggiormente gli "facevano onore". Questo gesto sarebbe, secondo Aretino, opera di alcuni "invidiosi" che "han tolto due lettere del primo libro de le mie, l'una che me ne manda il Vasto, l'altra che mi scrive Michelagnolo; [...] che il sì gran Capitano, e il sì degno scultore, mi sublimassero sì alto con le parole, prima ne hanno levato i detti che più mi facevano onore, e poi datele a imprimere a Paolo Manuzio in compagnia di molte altre" 38 . Le lettere citate si trovano effettivamente nella silloge dello stampatore umanista e precisamente nel secondo libro, dove sono collocate, a distanza di poche pagine una dall'altra, la lettera del marchese del Vasto, Alfonso d'Avalos, e quella di Michelangelo Buonarroti ${ }^{39}$. Ciò che si nota a prima vista è che, al contrario dell'abitudine di Aretino di inserire la data di ciascuna

36 A tale proposito vedi Fabio Massimo Bertolo, Aretino e la stampa, cit., pp. 30-31, 49.

37 Per le varie edizioni (e i nomi dei tipografi) del primo libro, cfr. Fabio Massimo Bertolo, Aretino e la stampa, cit., pp. 55-62.

38 Pietro Aretino, Lettere, Libro Terzo, cit., lett. 608, Venezia gennaio 1546, p. 463.

39 Paolo Manuzio (a cura di), Lettere volgari di diversi nobilissimi huomini et eccellentissimi ingegni scritte in diverse materie, cit., libro II, pp. 37, 44. 
lettera, le due epistole stampate dal Manuzio mancano di datazione (questa risulta, in generale, carente nel primo e solo in parte nel secondo libro per poi apparire con costanza). Tale mancanza, comunque, poteva rendere difficile, allora come oggi, individuare le lettere in questione all'interno del vasto epistolario aretiniano. Ricercando la prima edizione del secondo libro delle Lettere volgari e compiendo un raffronto tra i testi inseriti nella silloge del curatore-editore e quelli presenti nelle lettere scritte a Pietro Aretino, notiamo nella parte centrale delle variazioni minime (nella scrittura di certi termini). Manca però nell'intestazione della lettera del Marchese del Vasto il riferimento ad Aretino come "Al molto Magnifico Signor", mentre in quella di Michelangelo l'appellativo di "Divino" ("Al Divino Aretino") e infine l'espressione "il sempre vostro Michelagnolo" con cui il grande artista si congeda da Pietro Aretino ${ }^{40}$. La questione degna di attenzione non è tanto l'omissione di per sé, ma la scelta obbligata del "flagello dei principi" di non incolpare lo stimato tipografo veneziano, ma degli anonimi "invidiosi”" che avrebbero volutamente fornito a quest'ultimo delle lettere "incomplete". Certamente la mancanza di parti così significative poteva infastidire non poco Aretino, cosciente pure della notorietà che stava ottenendo la raccolta.

Le Lettere volgari, contenendo le epistole dei personaggi più in vista del periodo, riscossero, in effetti, sin da subito un enorme successo, tanto da richiedere al Manuzio una ristampa (con delle correzioni) già l'anno successivo; nel 1543 la raccolta uscirà nuovamente dalla sua tipografia, contrassegnata dalla ben nota marca aldina (l'àncora col delfino $)^{41}$. A questa seguiranno altre ristampe e dal 1542 al 1567 l'opera arriverà addirittura a ventotto edizioni, diventando l'antologia di lettere di maggior successo nel Cinquecento e, di conseguenza, anche la più imitata ${ }^{42}$. A tale proposito, Aretino dirà nel 1550 che il "Manuzio celeberrimo Paolo" è "oggi dì senza pari in le lettre",

40 La lettera del marchese del Vasto si trova in: Pietro Aretino, Lettere, Libro Primo, cit., lett. XXV (lettere diverse a l'autore), 20 novembre 1541, p. 492. Nell'epistola, Michelangelo rivela di non poter utilizzare nessuno dei suggerimenti forniti da Aretino poiché ha già portato a termine l'ideazione dell'affresco, ma loda comunque le sue idee asserendo: "se il dì del giudicio fusse stato, et voi lo aveste veduto in presentia, le parole vostre non lo figurarebbero meglio". Vedi Lettere scritte a Pietro Aretino, cit., lett. 396, 20 novembre 1537, p. 369. I testi sono stati paragonati con quelli presenti nella raccolta del Manuzio, Lettere volgari di diversi nobilissimi huomini et eccellentissimi ingegni scritte in diverse materie, Libro secondo, In casa de' figlioli di Aldo, Venezia 1545 (da differenziarla dall'edizione del 1558, da cui sono tratte le citazioni precedenti), pp. 40-41, 44-45.

41 Fabio Massimo Bertolo, Aretino e la stampa, cit., pp. 61-62.

42 Dato ripreso da Lodovica Braida, Libri di lettere. Le raccolte epistolari del Cinquecento tra inquietudini religiose e "buon volgare", Laterza, Roma-Bari 2009, pp. 7, 34. 
motivo per cui, nonostante i diversi tentativi da parte di altri curatori e tipografi, "nessuno esser gli può simile"43. Tra le raccolte epistolari posteriori, che sin dal titolo possono considerarsi una chiara imitazione dell'opera manuziana, si citeranno almeno le Lettere di diversi eccellentissimi huomini, raccolte da diversi libri, selezionate da Lodovico Dolce ${ }^{44}$. La silloge curata dal Dolce esce nel 1554 presso il tipografo veneziano Gabriele Giolito (lo stesso che aveva pubblicato il terzo libro di Aretino), ma non gode di una vasta diffusione e nel Cinquecento avrà una sola riedizione, nel $1559^{45}$. Nella parte introduttiva, Lodovico Dolce non può far a meno di annoverare il fatto che, oltre a lui, "una copia grandissima di scrittori" hanno seguito il modello proposto dal "dottissimo" tipografo veneziano ${ }^{46}$.

Quello che mancava alle altre raccolte era sicuramente la vastità dei contatti che possedeva invece Paolo Manuzio, come traspare dall'alto numero di lettere di autori diversi che era riuscito a reperire. $\mathrm{Da}$ alcune di queste emerge il meccanismo di ricerca attuato dal tipografo per ottenere nuove corrispondenze da stampare. Interessanti a tale proposito due lettere scritte da Claudio Tolomei e incluse nel secondo libro delle Lettere volgari di diversi nobilissimi huomini. Nella prima (datata 11 agosto 1543) il Tolomei rifiuta la richiesta del tipografo veneziano di far stampare le sue lettere (nonostante ammetta di averle suddivise in ben sette libri): "[...] non le condussi mai a quella finezza che bisognava, parte impedito da certe occasioni [...] Queste son, credo, quelle lettere che voi mi domandate, le quali (crediate a me) vivranno men dishonorate nelle tenebre che nella luce". Ritiene poi le sue lettere solamente delle "ciancie debili e sciocche" la cui pubblicazione non gioverebbe

43 Pietro Aretino, Lettere, Libro Quinto, a cura di Paolo Procaccioli, Salerno Editrice, Roma 2001, lettera 465, Venezia maggio 1550, p. 369. L'espressione "nessuno esser gli può simile" rivolta a Paolo Manuzio si trova in una poesia intitolata Ternali in gloria de la reina di Francia presente nel sesto libro delle Lettere. Pietro Aretino, Lettere, Libro Sesto, a cura di Paolo Procaccioli, Salerno Editrice, Roma 2002, lett. 32, Venezia 1550, v. 252, p. 49.

44 Anche nella raccolta di Lodovico Dolce compaiono lettere indirizzate a Pietro Aretino, tra queste ricordiamo un'epistola di Michelangelo (segnaliamo che non si tratta della stessa che compare nella raccolta di Paolo Manuzio). Cfr. Lodovico Dolce (a cura di), Lettere di diversi eccellentissimi huomini, raccolte da diversi libri, Gabriele Giolito, Venezia 1554, p. 377.

45 Per le edizioni dell'opera, cfr. Amedeo Quondam (a cura di), Le carte messaggiere. Retorica e modelli di comunicazione epistolare: per un indice dei libri di lettere del Cinquecento, Bulzoni Editore, Roma 1981, p. 281.

46 Cfr. Lodovico Dolce (a cura di), Lettere di diversi eccellentissimi huomini, cit., p. 4. Non solo il Dolce ha tratto ispirazione dall'iniziativa del Manuzio, ma ha ripreso un gran numero di lettere direttamente dalla raccolta di quest'ultimo. 
certo all'editore (espediente usato per evitare l'insistenza da parte di quest'ultimo $)^{47}$. Nella seconda lettera, invece, il Tolomei prega il tipografo di non includerlo nella propria raccolta o perlomeno di lasciargli modificare alcune sue epistole: "Di gratia M. Paolo, se egli è possibile, non mi fatte ingiuria di stamparlo e se pur non ve ne potete tenere, rimandatemele, vi prego, prima, perch'io le rivegga, e le ricorregga un poco"48. Queste corrispondenze ci permettono di approfondire il modo in cui il curatore-tipografo richiedeva il materiale da pubblicare, ma ci fanno pure comprendere le difficoltà incontrate nel selezionare le epistole a sua disposizione.

Paolo Manuzio, che aveva già incluso nella raccolta antologica delle lettere private, in seguito dedicherà alle proprie missive ben quattro libri intitolati semplicemente Lettere volgari (i primi tre usciti nel 1556, mentre il quarto nel 1560). Da quest'epistolario si nota chiaramente il vasto numero di relazioni strette dal tipografo veneziano con protagonisti della scena politica e stimati uomini di cultura ${ }^{49}$.

Con i libri di Lettere volgari del Manuzio ci troviamo davanti alla particolare scelta di un editore di raccogliere egli stesso un'antologia di lettere di autori diversi, ma anche quella di pubblicare separatamente le proprie corrispondenze. Per tale motivo, in questo genere, Paolo Manuzio costituisce un esempio di autore-curatore-tipografo che non ha precedenti nell'editoria italiana ${ }^{50}$.

Il Manuzio inserirà nel terzo libro anche una lettera indirizzata a Pietro Aretino che risiedeva a Venezia da quasi trent'anni ma che era ricorso alla tipografia aldina soltanto per le riedizioni di due opere sacre (nel 155152). Nell'epistola in questione, datata 3 febbraio 1555, il figlio di Aldo Manuzio esalta la grandezza del "flagello dei principi" riconoscendo la

47 Paolo Manuzio (a cura di), Lettere volgari di diversi nobilissimi huomini, cit., Libro secondo, p. 13. Al contrario di quanto riferito al Manuzio circa il proposito di non pubblicare la sua corrispondenza, i suoi sette libri di lettere usciranno nel 1547 presso la stamperia di Giolito de' Ferrari. Cfr. Amedeo Quondam (a cura di), Le carte messaggiere, cit., p. 314.

48 Paolo Manuzio (a cura di), Lettere volgari di diversi nobilissimi huomini, cit., Libro secondo, 21 febbraio 1545, p. 23.

49 Paolo Manuzio, Tre libri di lettere volgari di Paolo Manutio, In casa de' figlioli di Aldo, Venezia 1556, Libro I, 26 febbraio 1554; 3 marzo 1554, pp. 28-29.

50 Ricordiamo inoltre che il Manuzio è pure curatore di un'antologia di lettere di autori vari scritte in latino, Epistolae clarorum virorum, selectae de quamplurimis optimae, ad indicandam nostrorum temporum eloquentiam, uscita presso la sua tipografia nel 1556. Cfr. Tiziana Sterza, Paolo Manuzio editore a Venezia (1533-1561), cit., p. 146. 
sua proclamata originalità e opposizione ai principi dell'imitazione: "A voi non piacque giammai di porre il piede ove che apparissero l'orme di antico o di moderno scrittore", ritenendo tutte le sue opere frutto dell'“invenzione della mente vostra”. L'editore veneziano, sembra qui, indirettamente, far riferimento alla paternità aretiniana della stampa del primo libro di lettere d'autore in volgare, genere che aveva riscosso ampia fortuna anche nella sua tipografia ${ }^{51}$. Forse il riconoscimento maggiore della stima di Paolo nei confronti dell'originalità del "Divino" Aretino si può derivare dalla decisione di pubblicare la lettera appena citata nella riedizione del 1560 , nonostante fosse indirizzata a un autore la cui opera omnia era finita (l'anno prima) nell'indice dei libri proibiti52 .

Le considerazioni sui tipografi veneziani presenti nelle Lettere di Pietro Aretino e i riferimenti a quest'ultimo, e ad altri letterati, nelle raccolte e nelle corrispondenze private di Paolo Manuzio ci consentono di ricostruire, almeno in parte, i complessi meccanismi editoriali dell'epistolografia rinascimentale.

51 Si cita la lettera contenuta nell'edizione del 1560, cfr. Lettere volgari di M. Paolo Manutio divise in quattro libri, In casa de' figlioli di Aldo, Venezia 156o, Libro III, pp. 113-114.

52 Nel 1560, Paolo Manuzio non si limita a pubblicare tutte le lettere contenute nei primi tre libri, ma ne stampa alcune omettendo i nomi ritenuti compromettenti, mentre altre, probabilmente per precauzione, preferisce eliminarle; scelta necessaria anche perché in quel periodo "aspirava a diventare stampatore pontificio". Lodovica Braida, Libri di lettere. Le raccolte epistolari del Cinquecento tra inquietudini religiose e "buon volgare", cit., pp. 174-176. 


\section{BIBLIOGRAFIA}

AQUILECCHIA 1994

Giovanni Aquilecchia, Nuove schede di italianistica, Salerno Editrice, Roma 1994.

\section{ARETINO 1974}

Pietro Aretino, Lettera al Piccolomini, in Tutto il teatro, a cura di Antonio Pinchera, Newton, Roma 1974.

\section{ARETINO 1997}

Pietro Aretino, Lettere, Libro Primo, a cura di Paolo Procaccioli, Salerno Editrice, Roma 1997.

\section{ARETINO 1916}

Pietro Aretino, Il secondo libro delle lettere. Parte prima, a cura di Fausto Nicolini, Laterza, Bari 1916.

\section{ARETINO 1999}

Pietro Aretino, Lettere, Libro Terzo, a cura di Paolo Procaccioli, Salerno Editrice, Roma 1999.

ARETINO 2000

Pietro Aretino, Lettere, Libro Quarto, a cura di Paolo Procaccioli, Salerno Editrice, Roma 2000.

\section{ARETINO 2001}

Pietro Aretino, Lettere, Libro Quinto, a cura di Paolo Procaccioli, Salerno Editrice, Roma 2001.

\section{ARETINO 2002}

Pietro Aretino, Lettere, Libro Sesto, a cura di Paolo Procaccioli, Salerno Editrice, Roma 2002.

\section{ARETINO}

Pietro Aretino, In Laude di Venetia, in Scritti di Pietro Aretino nel Codice Marciano, It. XI 66 (=6730), D. Romei (a cura di), in http://www.nuovorinascimento.org/n-rinasc/testi/pdf/aretino/scritti.pdf.

BERTOLO 2003

Fabio Massimo Bertolo, Aretino e la stampa. Strategie di autopromozione a Venezia nel Cinquecento, Salerno Editrice, Roma 2003.

BRAIDA 2009

Lodovica Braida, Libri di lettere. Le raccolte epistolari del Cinquecento tra inquietudini religiose e "buon volgare", Laterza, Roma-Bari 2009.

DAMIANI 2016

Martina Damiani, Il confronto di approcci diversi alla minaccia straniera nelle commedie rinascimentali, in Comico e tragico nella vita del Rinascimento, Atti del XXVI Convegno Internazionale (Chianciano Terme- 
Pienza, 17-19 luglio 2014), a cura di Luisa Secchi Tarugi, Franco Cesati Editore, Firenze 2016, pp. 323-332.

DOLCE 1554

Lodovico Dolce, (a cura di), Lettere di diversi eccellentissimi huomini, raccolte da diversi libri, Gabriele Giolito, Venezia 1554.

FRANCO 1539

Nicolò Franco, Le pistole vulgari, Antonio Gardane, Venezia 1539.

LARIVAILLE 1987

Paul Larivaille, La vita quotidiana delle cortigiane nell'Italia del Rinascimento. Roma e Venezia nei secoli XV e XVI, Rizzoli, Milano 1987.

LARIVAILLE 1997

Paul Larivaille, Pietro Aretino, Salerno Editrice, Roma 1997.

MANUZIO 1545

Paolo Manuzio (a cura di), Lettere volgari di diversi nobilissimi huomini et eccellentissimi ingegni scritte in diverse materie, Libro secondo, In casa de' figlioli di Aldo, Venezia 1545.

\section{MANUZIO 1556}

Paolo Manuzio, Tre libri di lettere volgari di Paolo Manutio, In casa de' figlioli di Aldo, Venezia 1556.

\section{MANUZIO 1558}

Paolo Manuzio (a cura di), Lettere volgari di diversi nobilissimi huomini et eccellentissimi ingegni scritte in diverse materie, Domenico Giglio, Venezia 1558.

\section{MANUZIO 1560}

Paolo Manuzio, Lettere volgari di M. Paolo Manutio divise in quattro libri, In casa de' figlioli di Aldo, Venezia 1560.

\section{MARINI 2015}

Paolo Marini, Gli "Scritti" di Francesco Marcolini a cura di Paolo Procaccioli, in "Scaffale Aperto", VI, 2015, pp. 141-151.

PROCACCIOLI 2003

Paolo Procaccioli (a cura di), Lettere scritte a Pietro Aretino, Libro I, Tomo I, Salerno Editrice, Roma 2003.

\section{QUONDAM 1981}

Amedeo Quondam (a cura di), Le carte messaggiere. Retorica e modelli di comunicazione epistolare: per un indice dei libri di lettere del Cinquecento, Bulzoni Editore, Roma 1981.

\section{QUONDAM 1995}

Amedeo Quondam, Aretino e il libro. Un repertorio, per una bibliografia, in Pietro Aretino nel Cinquecentenario della nascita. Atti del Convegno di 
Roma-Viterbo-Arezzo (28 settembre-1 ottobre 1992), Toronto (23-24 ottobre 1992), Los Angeles (27-29 ottobre 1992), Tomo I, a cura di Enrico Malato, Salerno Editrice, Roma 1995, pp. 197-220.

STERZA 2008

Tiziana Sterza, Paolo Manuzio editore a Venezia (1533-1561), in Acme, Annali della Facoltà di Lettere e Filosofia Università Statale di Milano, vol. LXI, II, 2008, pp. 123-167. 


\section{RIASSUNTO}

\section{La centralità politico-culturale di Venezia negli scambi epistolari del primo Cinquecento}

Pietro Aretino intrattiene da Venezia diverse corrispondenze con personaggi di spicco del panorama politico e culturale, come traspare dai nomi altisonanti presenti nelle sue Lettere. Quando decide di rendere noti i suoi contatti epistolari e di includerli in un libro, ottiene un immediato successo che spinge il suo editore, Francesco Marcolini, a stampare nuove edizioni dell'opera. Con l'intento di ribadire il suo primato nell'epistolografia in volgare, Aretino biasima gli scrittori e i tipografi che pubblicano, subito dopo di lui, una moltitudine di libri di lettere, imitandolo. Dalla sua critica esonera però le persone più in vista di Venezia, tra cui Paolo Manuzio, prolifico autore ed editore di raccolte epistolari. Il presente lavoro si propone di analizzare quelle lettere che svelano il complesso meccanismo editoriale che sta dietro agli scambi epistolari del primo Cinquecento. 


\section{SAŽETAK}

\section{Središnji političko-kulturni položaj Venecije u epistolarnim razmjenama u prvoj polovici 16 . stoljeća}

Pietro Aretino održava iz Venecije razne korespondencije s važnim ličnostima iz političkih $\mathrm{i}$ kulturnih sfera, kao što je vidljivo iz uglednih imena prisutnih u njegovim Pismima (Lettere). Kada odluči obznaniti svoje epistolarne kontakte i uvrstiti ih u knjigu, odmah postiže veliku slavu, što će potaknuti njegovog izdavača, Francesca Marcolinija, na objavu novih izdanja toga djela. S nakanom da naglasi svoj primat $u$ epistolografiji na narodnom jeziku, Aretino osuđuje pisce i tiskare koji objavljuju, odmah nakon njega, veliki broj knjiga pisama, imitirajući ga. Kritike pošteđuje najuglednije članove venecijanskoga društva, među kojima Paola Manuzija, plodnoga pisca i urednika epistolarnih zbirki. U ovom radu analiziraju se pisma koja razotkrivaju složeni urednički mehanizam koji stoji iza epistolarnih razmjena u prvoj polovici 16. stoljeća.

Ključne riječi: knjige pisama, venecijanske tiskare, Pietro Aretino, Francesco Marcolini, Paolo Manuzio 